\title{
O ARQUIVO E A SOBREVIVÊNCIA DO DISCURSO: NOTAS SOBRE O CONCEITO DE "MUSEOLOGIA"
}

\author{
José Gomes Pinto \\ Universidade Lusófona de Humanidades e Tecnologias, Portugal \\ Carlos Pimenta \\ Universidade Lusófona de Humanidades e Tecnologias, Portugal
}

\begin{abstract}
Resumo: Procuramos tematizar o problema do arquivo como conceito constitutivo da possibilidade de se determinar o que é de valor patrimonial, museístico. Este conceito surge lançado sempre entre a disputa entre o sagrado e o profano, entre o novo e o velho, entre o que tem valor e dele é destituído. A partir destas aporias procuramos estabelecer as consequências que sobrevêm de se fazer esta curva reflexiva sobre determinados objectos patrimoniais. Esta tensa dialéctica é o que permite que certas obras culturais, quando aparecem como novas e profanas, que certas peças artísticas, por exemplo, entrem dentro das instituições museistícas, que certas peças literárias, entrem dentro da literatura, certas composições musicais, dentro da história da música, etc. Porque são enunciados que produzem sentido, que expressam a subjectividade e o mundo, o real.
\end{abstract}

Palavras-chave: arquivo; signo; suspeita; media; património.

\section{O arquivo e a sobrevivência do discurso}

Sabemos que a filosofia moderna surge como uma actividade que procura pensar as superfícies onde os signos fluem e formam um conjunto de sentidos a que chamamos realidade. Quando esses signos já não remetem a nada exterior, a não ser quando são descritivos, legítimos na sua verificabilidade, então o problema de determinação do que é real reside nos espaços onde os signos fluem e se reúnem para formar um todo com sentido e se mostrarem como efectivos ${ }^{1}$. Esses espaços denominam-se arquivos. $\mathrm{O}$ problema, pois, de que todo o pensamento contemporâneo que esteja preocupado com os sentidos que correm nos media, essas superfícies onde os signos fluem e se retêm, se transmitem e se armazenam, deverá ter em consideração a estrutura e significado do arquivo. Diz Boris Groys: «no arquivo coleccionam-se e custodiam-se coisas que são

\footnotetext{
${ }^{1}$ É interessante notar que em língua alemã, efeito, Wirkung tem a mesma raiz que Wirklichkeit, realidade, que no uso corrente pode significar verdade. Benard Bourgeois no seu Le vocabulaire de Hegel, traduz mesmo o conceito Wirklichkeit em Hegel por effectivité. Uma tradução arrojada, mas que corresponde ao espírito da letra (Bourgeois, 2011, 47).
} 
importantes, relevantes ou valiosas para uma determinada cultura; todas as outras coisas sem importância, irrelevantes ou sem valor, ficam fora do arquivo, no espaço profano» (Groys, 2008: 11). Neste sentido o que caracteriza o pensamento moderno é exactamente o ter-se dado conta de que o que importa ao homem e à vida é o que está dentro do arquivo. O espaço profano que está fora do arquivo carece de existência. $\mathrm{O}$ novo da modernidade é, justamente, ter-se dado precatado de que o que realmente importa está no comércio dos signos e no seu armazenamento: sejam eles museus, bancos de dados informáticos, a literatura, a música ou em geral a História, o passado, o já acontecido.

Essa tensão desenha-se no exemplo que Ezra Pound nos conta no seu $A B C$ of Reading:

No man is equipped for modem thinking until he has understood the anecdote of Agassiz and the fish:

A post-graduate student equipped with honors and diplomas went to Agassiz to receive the final and finishing touches. The great man offered him a small fish and told him to describe it.

Post-Graduate Student: 'That's only a sunfish.'

Agassiz: 'I know that. Write a description of it.'

After a few minutes the student returned with the description of the Ichthus Heliodiplodokus, or whatever term is used to conceal the common sunfish from vulgar knowledge, family of Heliichtherinkus, etc., as found in textbooks of the subject. Agassiz again told the student to describe the fish. The student produced a four-page essay. Agassiz then told him to look at the fish. At the end of three weeks the fish was in an advanced state of decomposition, but the student knew something about it (Pound, 1991: 17-18).

O brilhante estudante procura imediatamente nos arquivos o sentido daquilo que lhe foi pedido. Não podia estar noutro sítio a informação que era importante para conhecer algo que já alguém tinha descrito. O real, o exterior do arquivo, só adquire importância pela sua relação ao arquivo. Tudo o que fica fora do arquivo é insignificante quando comparando com todo o sentido que o arquivo contém. O peixe que o estudante tinha de observar, funciona aqui como um exemplo completo da superfície mediática: nela fluem signos que não remetem necessariamente ao seu exterior, mas apenas ao interior do próprio arquivo, do já conhecido. Só quando o peixe morre, pôde o estudante chegar a conhecer algo do peixe, porque o peixe deixou de ser essa superfície mediática onde a corrente de signos se proporciona ao espectador. Só a sua morte lhe permitiu dar-se conta do fora do arquivo, mas ainda sim, já tarde, aprender alguma coisa. Funciona como o Quadro negro de Kazimir Malevich: só quando percebemos que nada podemos pintar nele, porque já está coberto de tinta negra que impede a fixação de toda a 
representação pictorial, de toda a cor, é que cobra sentido para nós a actividade da pintura: «O Quadro negro não se mostra meramente como um quadro entre outros, mas como uma repentina revelação do suporte pictórico oculto, que se manifesta, com uma evidência esmagadora, no meio do mundo habitual e superficial das imagens e, como consequência do uso da violência por parte do artista» (Groys, 2008: 136-137). O mesmo veio a acontecer mais tarde no cinema quando Guy Debord com Hurlements en faveur de Sade, de Junho de 1952, apresentou numa sala de cinema um filme com projecções em negro e branco e com grandes espaços de silêncio, fazendo dizer a uma das vozes, a quinta: «Le cinéma est mort. Il ne peut y avoir de film, passons, si vous voulez, au débat» (Debord, 1994: 11). Mas o mesmo aconteceu com a apresentação da peça 4'33” de John Cage, onde era o silêncio que imperava e não o conjunto harmónico das notas musicais. O moderno, assim, é uma constante referência ao dentro do arquivo, ao dispositivo que regula a instituição da experiência. É um movimento que não tem por objectivo identificar o que é, a natureza das coisas, mas que procurar abrirse, estar no domínio do aberto, ao que vem de novo. É assim que o pensamento moderno se conecta mais com o capital - porque é este que regula o comércio que constitui o arquivo - e com a crítica, enquanto procura pensar as estruturas que possibilitam esse comércio de signos. Foi assim que Clement Greemberg viu o papel da pintura moderna:

Realistic, illusionist art had dissembled the medium, using art to conceal art. Modernism used art to call attention to art. The limitations that constitute the medium of painting -the flat surface, the shape of the support, the properties of pigment- were treated by the Old Masters as negative factors that could be acknowledged only implicitly or indirectly. Modernist painting has come to regard these same limitations as positive factors that are to be acknowledged openly (Greenberg, 1982: 6).

O que faz da modernidade moderna é o facto de atentar ao novo, de estar sempre numa posição de abertura e da crítica dos discursos contidos, imanentes, aos arquivos. Mas o novo não é uma coisa do agora, do presente, ainda que no presente se faça sentir de uma forma mais pesada, mas também menos pensada. O novo é um conceito que se com raízes no tempo. A constituição do arquivo é temporal no sentido em dentro dele se determina o que já é conhecido, o que ainda não é conhecido e que pode vir a ser conhecido. No arquivo faz parte o passado, o morto, mas a vocação do arquivo é a de eternizar o que dentro dele cai e portanto tem a vocação da conquista do tempo, como nos recordava Harold A. Innis. O actual, o vivo, o novo não cabe dentro dele, mas há arquivo essa pretensão à infinita duração, à eternidade também. Este é sempre uma promessa de tudo armazenar. 
Essa oposição que se traça dentro do arquivo entre o já conhecido e o por-vir, entre o antigo e o novo é aquilo que produz desconfiança no actual, no presente, no sem figura. O novo prende-se com as dificuldades inerentes do pensar, das formas de categorização humana. O novo vem sempre dado por formas de mediação artificial - a escrita, a pintura, a música - ou surge naturalmente do encontro do homem com o mundo. Quando se fala do novo na sua forma de expressão semântica, estamos quase sempre a falar de mediações, de novos sentidos que se inscrevem nos media ou de novos media que inscrevem formas outras de sentido, como acontece no discurso actual sobre a nossa condição histórica. Porque a todo o medium corresponde duas funções: a de arquivar e a de transmitir. Os instrumentos técnicos humanos vão armazenando dados que se tornam, por essa via, conhecidos, mas vão também produzindo outras formas de sentido que sobre eles correm. Quando transmitem, alargam o espaço e o tempo, aumentando por isso o alcance do já conhecido. Mas criam também camadas de sentido que se produzem sobre eles, porque, mesmo com a escrita, onde todos escrevem para todos e sobre os discursos de todos se produz sentido a partir do sentido anteriormente dado: na pintura, na música, na literatura, etc. Há exemplos em que essa produção tenta superar essa característica essencial de todos os media, que é a de armazenarem, dando a sensação de infinitude exterior, porque tudo pode ser armazenado pelos meios conquanto tudo é produzido sobre eles: superfícies onde os signos fluem.

A aparência de algo infinito que carrega o arquivo, tenta por a salvo o carácter instrumental do processo de armazenamento, superando assim a realidade finita dos media. Estes são sempre coisas físicas e como tais estão sujeitos às leis temporais da extinção, às catástrofes naturais, à vontade humana. Um quadro de Paul Cezanne só é tomado por eterno porque pertence a um arquivo com um discurso determinado. Desde o ponto de vista da sua materialidade, é finito. Não fosse a efectividade do arquivo, na conservação das peças artísticas - o museu -, e estes desapareceriam indelevelmente.

A forma do arquivo é por isso sempre uma actividade de conquista do tempo. Todos estes dispositivos funcionam como mecanismos que criam a sensação de infinitude, da qual procede o novo e do qual ele se resguarda da sua função de outro, da estranheza e por tanto também do medo.

Ora um arquivo, uma superfície mediática, enquanto transmite, armazena e espalha, deve manter-se unido e isso só se consegue se mantiver coeso, como um todo homogéneo de elementos que possuem algo de idêntico. Está a razão pela qual se afirma que o pensamento moderno o pensamento da diferença. Esta é razão, também, pela qual toda a forma de arquivamento, que é também toda a forma cultural, aponte 
para o espaço fora do arquivo e se designe este somente por anulação: o que fica fora do arquivo é o profano. É assim que Boris Groys formula o problema: «a economia do novo pressupõe uma diferença segura e estável entre o arquivo do culturalmente valioso e o espaço profano extra-cultural» (Groys, 2008: 19)². Quando na peça de teatro Huis Clos, que em português se traduziria À porta fechada ou Sem saída, de Jean-Paul Sartre, se afirma que o inferno são os outros (Sartre, 2000: 93)3, o que está a fazer é justamente descolocar o olhar do sujeito para com o mundo, para colocar o olhar numa duplicidade inusitada: o outro é o inferno porque é desde a posição do outro que compreendemos a nossa própria subjectividade, o nosso próprio eu. Criam-se assim um conjunto de visões, de modos de ver, que deslocam o ponto de vista. Descolocamos o olhar sobre o mundo, para o ponto de vista de um olhar sobre nós, mas que é de todo em todo produzido também por nós, pelo arquivo que perpetuamos. O arquivo enquanto realiza a operação do olhar, funciona como um espelho: aponta para uma exterioridade espectral. $\mathrm{O}$ arquivo é da ordem do fantasmagórico.

O outro é assim esse espaço profano que nos olha e que concionada o próprio entendimento que o homem faz de si mesmo, como se este, as subjectividades, estivessem de fora, no espaço profano. De resto, o que a alteridade vem trazer é sempre esse espaço novo, que não conseguimos controlar, porque a superfície onde se joga o sentido daquilo que pode ser tido como realidade, mas também de desejo, está marcado pela opacidade: tem-se medo do outro porque este é opaco, não transparente, porque enquanto superfície de signos, deixa sempre entrever que algo está por detrás e que não se consegue apreender, que sua abertura lhe pertence como uma questão de vontade. $\mathrm{O}$ discurso sobre a subjectividade arquivada na história do Ocidente, remete ao outro como um modo de nos olharmos: «sob o olhar do outro, envergonhamo-nos do nosso corpo, do nosso aspecto, do nosso comportamento, da imagem inteira com que nos

\footnotetext{
${ }^{2}$ É assim que Giorgio Agamben coloca o problema da profanação: «Los juristas romanos sabían perfectamente que significaba "profanar"... Sagradas o religiosas eran las cosas que pertenecían de algún modo a los dioses. Como tales, ellas eran sus traídas al libre uso y al comercio de los hombres, no podían ser vendidas ni dadas en préstamo, cedidas en usufructo o gravadas de servidumbre. Sacrílego era todo acto que violara o infringiera esta especial indisponibilidad, que las reservaba exclusivamente a los dioses celestes (y entonces eran llamadas propiamente "sagradas") o infernales (en este caso, se las llamaba simplemente "religiosas"). Y si consagrar (sacrare) era el termino que designaba la salida de las cosas de la esfera del derecho humano, profanar significaba por el contrario restituirlos al libre uso de los hombres. "Profano -escribe el gran jurista Trebacio - se dice en sentido propio de aquello que, habiendo sido sagrado o religioso, es restituido al uso y a la propiedad de los hombres". Y "puro" era el lugar que había sido desligado de su destinaci6n a los dioses de los muertos, y por lo tanto ya no era mas "ni sagrado, ni santo, ni religioso, liberado de todos los nombres de este genero" (D. 11, 7, 2). Pura, profana, libre de los nombres sagrados es la cosa restituida al uso común de los hombres. Pero el uso no aparece aquí como algo natural: a el se accede solamente a través de una profanación. Entre "usar" y "profanar" parece haber una relación particular, que es preciso poner en claro». (Agamben, 2005: 97-98). 3 Diz assim o texto original: «Alors, c'est ça l'enfer. Je n'aurais jamais cru ... Vous vous rappelez : le soufre, le bûcher, le gril... Ah! quelle plaisanterie. Pas besoin de gril: l'enfer, c'est les Autres» (Sartre, 2000: 93).
} 
mostramos ao mundo. A mera suspeita de que o olhar alheio me observe é suficiente para provocar essa vergonha universal»(Groys, 2008: 104). Ao fim de contas, se tomarmos o corpo como a superfície onde correm dos signos, a subjectividade é sempre algo que não se esgota nesse comércio de signos, dos gestos e dos desejos que os manifestam. O outro é o inferno, porque é tomado, justamente, como o profano, como o que está fora do arquivo, o novo, o insondável, aquilo que não tem figura de visibilidade, mas também pode ser que, sob esse efeito de espelho, nos olhemos a nós próprios como o novo, como o que está fora do arquivo, dos discursos, envergonhandonos assim desde o olhar dos outros.

O mesmo acontece com qualquer outra superfície mediática: a tela, o texto, a televisão, a rádio, o cinema. Não chegamos nunca a poder desmontar a estrutura e a referência do jogo de signos que correm sobre estes, como não conseguimos desmontar a carne que serve a superfície onde correm os signos que constituem a subjectividade. Só conhecemos o sujeito quando este estiver morto, só saberemos o que é a pintura quando a tivermos desarticulado, só saberemos o que é a televisão quando a pararmos, a desmontarmos e procurarmos saber como esta funciona, como com o cinema, como com a rádio, como o peixe do discípulo de Agassiz. Procurar uma explicação científica sobre todos estes suportes de fluências de signos é um exercício sem fim, porque enquanto eles forem suportes, ficarão por determinar, deixando apenas ver o que sobre eles desliza. Se quiséssemos ver o que possibilita esse comércio de signos, teríamos de apagar o pintado - os conjunto de signos que sobre esse medium se apresenta -, teríamos de retirar as letras, para ver o papel, desmontar a televisão para ver como esse comércio é ali possibilitado, teríamos de desmontar o celulóide e reconstruir a montagem do filme, para saber com se processou o sentido que nele se apresentou. Esse processo de desmontagem, de desconstrução, permanece sempre como desejo, porque o que está fora do arquivo, de cada um enquanto subjectividade, da pintura enquanto lá está o pintado, da televisão enquanto lá está o representado, é justamente aquilo que fica sempre de impensado, de invisível. Toda a estrutura da mediação permanece opaca, mas é essa justamente a sua essência: a de se mostrar transparente, a de se anular no modo de apresentar o comércio de signos que sobre ela correm. Tem assim razão Marshall McLhuan quando nos diz que «na verdade até bastante sintomático que o conteúdo de qualquer meio nos impeça de entrever a natureza desse mesmo meio» (McLuhan, 2008: 22). Da mesma forma que acertam Jay David Bolter e Richard Grusin ao afirmarem em Remediation. Understanding New Media que a finalidade de toda a estrutura de mediação, pelo menos desde o seu ponto de vista 
físico, é a criar a ideia de imediação e de transparência (Bolter \& Grusin, 2000: 70)4. Ou o que é o mesmo, de se ocultarem enquanto estrutura físicas de mediação. Só a corrente de signos que flui por cima dos media tem assim importância, levando a uma lógica da imediação que aparentemente deixa o espectador no papel de crente, ou seja, de que aquilo que se está realmente a passar diante dele é o 'real' ou outro dele mesmo - que é outra forma de dizer o 'real' -, internalizando-se assim como real e passando a fazer parte do reportório, do arquivo, das coisas que cabem dentro do arquivo e podem passar ser tomadas como existentes e, por isso, sagradas.

O novo fica assim protegido sob a capa do imediato, que remete sempre a uma forma já arquivada e esta constitui o real, pois só é real tudo o que ainda não está em arquivo, mas que pode já pertencer a uma lógica do próprio arquivo: «A chamada realidade não é, no fundo, mais que a soma de tudo aquilo que ainda não foi recolhido pelos arquivos. Por isso a realidade não é algo primário que tivesse sido representado no espaço secundário do arquivo; a realidade é tudo aquilo que ficou fora do arquivo» (Groys, 2008: 13). O real, o novo, somente pode ser recolhido no arquivo se passar a fazer parte dessa crença que o faz tomar-se como sagrado: como algo que pode ser digno de pertencer ao arquivo. O real, visto desde o seu sentido histórico, pertence sempre ao domínio do milagre, do dom, do aberto. Se o real fosse o que pode ser descrito cientificamente, os facta científicos, então nada se poderia esperar. Mas faz parte da definição do real a promessa de devir, de ser outro.

A grande disputa política sobre o arquivo joga-se justamente aí: o como é que se pode ou o que é que deve ser tomado por real, o novo, para que possa caber dentro do arquivo? Para Wittgeinstein era justamente a linguagem: a corrente signíca. Mas esta só se faz sobre uma superfície, os media. Esta tensa dialéctica é o que permite que certas obras culturais, quando aparecem como novas e profanas, que certas peças artísticas, por exemplo, entrem dentro das instituições museistícas, que certas peças literárias, entrem dentro da literatura, certas composições musicais, dentro da história da música, etc. Porque são enunciados que produzem sentido, que expressam a subjectividade e o mundo, o real.

Mas este fenómeno de ameaça do novo é já clássico. Quando Joseph Addison em Os prazeres da imaginação (Addison, 1991) 5 apresenta a figura do novo, da novidade

\footnotetext{
4 «We have so far used the term immediacy in two senses: one epistemological, the other psychological. In the epistemological sense, immediacy is transparency: the absence of mediation or representation. It is the notion that a medium could erase itself and leave the viewer in the presence of the objects represented, so that he could know the objects directly. In its psychological sense, immediacy names the viewer's feeling that the medium has disappeared and the objects are present to him, a feeling that his experience is therefore authentic». (Bolter \& Grusin, 2000: 70).

5 «Todo lo que es nuevo o singular da placer a la imaginación; porque llena el ánimo de una sorpresa
} 
como o motor tanto do conhecimento como do prazer que se possa despertar na mente humana, antecipa e possibilita aquilo que mais tarde David Hume e Edmund Burke vieram a dizer sobre o mesmo problema. As reflexões destes pensadores, ainda que pareçam extemporâneas, assumem grande importância para a compreensão da actualidade, porque é este um tempo onde o novo se apresenta na sua forma mais acutilante e onde as superfícies mediáticas mais sentido produzem no real e este é tomado na sua forma empírica e não signica. A crítica feita pela filosofia empirista é, hoje, verdadeiramente actual. Para estes pensadores do século XVII, a novidade apresenta-se sempre como uma figura para a qual não há ainda experiência, porque o novo se constitui como algo que está para além de todo o conhecimento e este, naturalmente, deve ser considerado como uma forma histórica da arquivar a verdade e, por isso, o real. O novo aparece como aquilo que desperta uma experiência para a qual ainda não há forma de enquadramento, que permanece por tanto como o impensado, como fora de toda a possibilidade de reconhecimento. Como milagre, se quisermos utilizar as palavras de Wittgenstein. Apresenta-se assim como aquilo que causa medo, incerteza, dúvida e suspeita, ainda que necessariamente deva despertar também a curiosidade, o prazer, que para alguns é rápido, e que advém justamente dos hiatos encontrados para o seu reconhecimento. O novo, se cria medo, desperta concomitantemente a necessidade de aumentar o conhecimento, de alagar o arquivo. Porque todo o arquivo se sabe sempre - este é o duplo paradoxo do arquivo -, como incompleto, mas cujo desejo é o da completude: assusta aquilo que não está dentro dele, o real, o empírico na sua forma mais crua, a da individualidade dos acontecimentos. A suspeita torna-se legítima, em vista do conhecimento passado, mas abre ao mesmo tempo a promessa de poder vir a ser aumentado, aumentando também, por via da superação, a sensação de segurança, de conhecimento, de familiaridade. $O$ filósofo escocês mais comprometido com a modernidade, David Hume, foi um dos primeiros a pensar o problema epistemológico e o paradoxo que contém o problema do novo e por tanto do arquivo e da suspeita que este levanta. Diz no Tratado da natureza

agradable; lisonjea su curiosidad; y da la idea de cosas que antes no había poseído. Estamos en verdad tan familiarizados con cierta especie de objetos, y tan empalagados con la repetición de unas mismas cosas, que todo cuanto sea nuevo o singular contribuye no poco a diversificar la vida, y a divertir algún tanto el ánimo con su extrañeza: porque ésta sirve de alivio a aquel tedio de que nos quejamos continuamente en nuestras ordinarias y usuales ocupaciones» (Addison, 1991: 140). Diz o original: « «Every thing that is new or uncommon raises a Pleasure in the Imagination, because it fills the Soul with an agreeable Surprize, gratifies its Curiosity, and gives it an Idea of which it was not before possest. We are indeed so of- ten conversant with one Set of Objects, and tired out with so many repeated Shows of the same Things, that whatever is new or uncommon contributes a little to vary human Life, and to divert our Minds, for a while, with the Strangeness of its Appearance: It serves us for a kind of Refreshment, and takes off from that Satiety we are apt to complain of in our usual and ordinary Entertainments. It is this that bestows Charms on a Monster, and makes even the Imperfections of Nature please us. It is this that recommends Variety, where the Mind is every Instant called off to something new, and the Attention not suffered to dwell too long, and waste it self on any particular Object. It is this, likewise, that improves what is great or beautiful, and make it afford the Mind a double Entertainment». (http://philosophy.lander.edu/intro/articles/addisonart-a.pdf.) 
humana:

É legitimo duvidar de tudo o que ainda não tem figura estável, daquilo que não tem conceito no qual possamos enquadrar o que aparece e assim reconhecer o que aí se apresenta. O repentino e o estranho de uma aparição produz naturalmente uma comoção na mente, de igual modo a que se produz com tudo aquilo que não estávamos acostumados nem preparados. Por outro lado, esta comoção produz naturalmente a curiosidade e o desejo de saber que, ao ser muito violento por causa de um violento e repentino impulso do objecto, resulta inquietante, assemelhandose na sua agitação e incerteza à sensação de medo ou a paixões compostas de tristeza e alegria. [...] Deste modo, todas as classes de incerteza têm uma conexão forte com o medo, ainda quando não produzam qualquer oposição de paixões mediante as imagens ou de considerações opostas que se nos apresentam» (Hume, 1739: 446).

Edmund Burke, por outro lado, preferia sublinhar o carácter superficial da novidade e da curiosidade que esta desperta: «mas aquelas coisas que nos subjugam meramente pela sua novidade, não podem reter-nos muito tempo, a curiosidade é o mais superficial dos afectos» (Burke, 1997: 23). Esta dupla forma em como os filósofos empiristas resolveram o problema, joga-se precisamente nesta noção de arquivo. Para os cépticos, aqueles que fazem da suspeita um modo de aproximação ao real, o que está em jogo é justamente uma descrença nesses elementos constitutivos dos arquivos imateriais e transcendentes que caracterizaram os platonismos, o cristianismo, o cartesianismo e depois, a filosofia absolutista de Hegel. Se fazemos da suspeita o princípio motor, estamos sempre na atitude de questionar o arquivo, o já conhecido, mas também na atitude de abertura para com o novo, ainda que esta se observe, por necessidade da fragilidade do património transcendental, ao mesmo tempo com receio e com esperança.

O que sucede hoje, num mundo que está totalmente mediatizado, é que se tenta fazer perder esta dupla atitude de abertura: por um lado de questionamento do arquivo e por outro, de abertura ao fora do arquivo, de forma a poder reformulá-lo e acrescê-lo de novos elementos. O pensador céptico não se pode caracterizar como sendo um pensador cauto, mas como sendo um pensador lúcido, vigilante: atenta aos critérios do processo de armazenamento de informação, mas ao mesmo tempo está na disposição de a re-ordenar. Essa reordenação do arquivo é uma tarefa técnica, ainda que dentro dela surjam, em paralelo, os problemas que as grandes teorias absolutistas criaram: a da oposição ser e aparência, certeza e dúvida, ou por outras palavras, exterior e interior do património cultural, do arquivo. 
Todo o arquivo é, como nos diz Derrida, hipomnémico ${ }^{6}$, quer isto dizer que todo o arquivo é do domínio técnico. É dispositivo elaborado com o fim de auxiliar as formas de enquadramento do real. Todo o arquivo é instrumento da memória. Enquanto arquivo se refere a princípio, à arkhê grega, remete para a lei original7. O arquivo é a base económica da memória, a forma mais segura de a gerir. O que fica arquivado pertence ao arquivo apenas na medida em que este remete para algo distinto dele mesmo, para algo que lhe é exterior ${ }^{8}$. O arquivado anula-se, constantemente a si mesmo, para ceder lugar àquilo ele próprio exclui: o novo, a sua conexão com o tempo futuro, o real, o por-vir9: «se quisermos saber o que o arquivo teria querido dizer, não o saberemos mais que no tempo por vir. Talvez. Não amanhã, mas no tempo por vir, brevemente ou talvez nunca ${ }^{10}$. Nele reside sempre uma promessa ainda não cumprida, ainda por cumprir. O arquivo, foi neste sentido que pensámos a afirmação de Sartre, é sempre um instrumento que dirige o olhar, diz Derrida:

...este algum outro espectral olha-nos, sentimo-nos olhados por ele, fora de toda a sincronia, antes inclusive e mais além de todo o olhar da nossa parte, conforme a uma anterioridade (que pode ser da ordem da geração, de mais de uma geração) e a uma dissimetria absolutas, conforme a uma desproporção absolutamente indominável. A anacronia dita aqui a lei. O efeito de viseira desde o qual herdamos a lei é isso: o sentir-nos vistos por um olhar com o qual será sempre impossível cruzar o nosso. (...) submissão essencialmente cega ao seu segredo, ao segredo da sua origem: primeira obediência à injunção, que condicionará todas as outras (Derrida, 1995: 21-22).

Na superfície mediática onde os signos correm, onde o mundo se expressa, mas não consegue reconhecer o seu referente, conhece o arquivo a sua atitude de princípio: a da

\footnotetext{
6 «Como impulso de morte é, também, segundo as palavras mais destacadas do próprio Freud, um impulso de agressão e de destruição (Destruktion), ela não só empurra ao esquecimento, à amnésia, à aniquilação da memória, como mnéme ou anamnése, mas ordena a si mesma o apagamento radical, a erradicação na verdade, do que jamais se reduz à mnéme ou à anamnése, a saber, o arquivo, a consignação, o dispositivo documental o monumental como hypomnema, suplemento ou representante mnemotético, auxiliar ou memorando». (Derrida, 1996: 19).

7 Derrida é explícito: «Não comecemos pelo começo, nem sequer pelo arquivo. Mas sim pela palavra «arquivo»-y pelo arquivo de uma palavra tão familiar. Arkhé, recordemos, nomeia ao mesmo tempo o começo e o mandato. Este nome coordena aparentemente dois princípios em um: o principio segundo a natureza ou a história, ali onde as coisas começam-principio físico, histórico ou ontológico-, mas também o princípio segundo a lei ali onde os homens e os deuses mandam, ali onde se exerce a autoridade, a ordem social, nesse lugar desde o qual a ordem é dada-princípio nomológico». (Derrida, 1996: 9)

8 «...assim a escrita, o vestígio, a inscrição sobre um suporte exterior ou sobre o corpo chamado próprio como por exemplo -e este não é um exemplo qualquer para mim- esse arquivo singular e imemorial que se chama circuncisão e que pelo facto de já não nos abandonar nunca, não por isso resulta menos sobrevindo e exterior, exterior em pleno corpo próprio». (Derrida, 1996: 34)

9 «'arquivo' é apenas uma noção, uma impressão associada a uma palavra e para a qual nem Freud nem nós temos conceito algum». (Derrida, 1996: 37).

10 (Derrida, 1996: 44).
} 
suspeita. Suspeitamos sempre que há algo por detrás do aparecer, mas esse aparecer já não é mais o aparecer espontâneo, voluntário da natureza, que se mostra e esconde constantemente. Já não é da ordem da dialéctica clássica, de que no aparecer se esconde uma essência, de que o pensamento tende a dirigir-se e a expressar essa essência do aparecer, de que o visível esconde um invisível que deve ser desocultado. É isto que distingue dúvida de suspeita: porque o que cancela a dúvida é da ordem do involuntário. Posso começar por suspeitar de todas as minhas crenças, de todos os conhecimentos que já possuo, que estão dentro do arquivo, com o fez Descartes, posso suspender toda a nova aparência e tomá-la como falsa aparência, posso levantar a hipótese hiperbólica de um ente que tenha a capacidade de controlar os meus pensamentos e me fazer errar em todos eles. Tudo isso eu posso fazer com a suspeita. Mas a suspeita não tem cura, como a dúvida. A dúvida pode ser resolvida pela perceptio clara et distincta, por uma resposta não mediatiza que só encontra na revelação a sua justificação. A revelação é a origem do fim da dúvida, da mesma forma que numa experiência laboratorial ao cientista se lhe apresenta o facto que comprova a sua suspeita, resolvendo-se assim a dúvida. Mas a suspeita enquanto principio motor do arquivo não tem qualquer resposta ou resolução fora das estruturas da mediação. Se o comércio de signos corre sobre as superfícies mediáticas e estas são sempre estruturas técnicas, não existe qualquer possibilidade de que estas se nos revelem, já que a sua função é a de serem justamente o espaço de fluxo do sentido. O que os pósestruturalistas descobriram foi justamente a morte do autor, a morte do homem, ou seja, de que não há um sujeito único por detrás dos discursos, porque estes são correntes de signos que funcionam sobre superfícies técnicas e são construídos social e historicamente. Nietzsche tinha já declarado a morte de Deus, Malevich fê-lo com a pintura, Duchamp com o sistema da arte, Cage com a música, Debord, talvez, com o cinema. Mas ainda assim todos estes discursos sobreviveram. A razão da sua sobrevivência está no arquivo. Eles ainda conseguem ser significativos, ainda que necessitem da citação para se apresentarem como tal. Já não dizemos Deus, mas sim «como alguém disse, Deus...» já não falamos da arte como referente, mas usamos a citação para falar dela. Deslocamo-nos sobre a superfície mediática para interrogar o sentido, sabendo que não vamos encontrar nada que consiga parar essa suspeita. O que encontramos então? Apenas uma corrente de signos. 


\section{Referências Bibliográficas}

Addison, Joseph (1991) Los placeres de la imaginación y otros ensayos de «The Spectator». Madrid: Visor.

Agamben, Giorgio (2005) Profanaciones. Buenos Aires: Adriana Hidalgo.

Bolter, Jay David \& Grusin, Richard (2000) Remediation. Understanding New Media. Cambridge/Mass.: MIT Press.

Bourgeois, Bernard (2000) Le vocabulaire de Hegel. Paris: Ellipsis.

Burke, Edmund (1997) Indagación filosófica sobre el origen de nuestras ideas acerca de lo sublime y de lo bello. Madrid: Tecnos.

Debord, Guy (1994) Oeuvres cinématographiques complètes. 1952-1978. Paris: Gallimard.

Derrida, Jacques (1995) Espectros de Marx. Madrid: Trotta.

Greenberg, Clement (1982) «Modernist Painting», in Frascina, Francis \& Harrison, Charles (ed.) Modern Art and Modernism: a Critical Anthology. New York: Harper and Row.

Groys, Boris (2008) Bajo sospecha Una fenomenología de los medios. Valencia: PreTextos.

Hume, David (1739) A Treatise of Human Nature: Being an Attempt to Introduce the Experimental Method of Reasoning into Moral Subjects. Reprinted form the original edition in three volumes and edited with an Analytical Index by Selby-Bigge, L.A. Oxford: Oxford at the Clarendon Press, (1739) 1888 (Second edition with text revised and notes by P. H: Nidditch, 1975 .

McLuhan, Marshall (2008) Compreender os meios de comunicação. As extensões do homem. Lisboa: Relógio d’Água.

Pound, Ezra (1991) ABC of Reading. London \& Boston: Faber and Faber.

Sartre, Jean-Paul (2000) Huis Clos. Suivi de "Les mouches”. Paris: Gallimard. 
José Gomes Pinto é professor associado na Escola de Comunicação, Arquitectura, Artes e Tecnologias da Informação (ECATI) da Universidade Lusófona de Humanidades e Tecnologias. Licenciado em Filosofia, obteve um doutoramento em Estética e Teoria das Artes financiado pela FCT. Fez estudos de pósdoutoramento na universidade Humboldt de Berlin. Colaborou em diversos projectos de investigação em Portugal e em Espanha. Dirigiu e criou os cursos de licenciatura em Comunicação e Artes e o mestrado em Produção e Criação em Artes Tecnológicas. Actualmente é subdirector da ECATI, dirigindo também os cursos de terceiro ciclo em Filosofia - Especialidade Pensamento Contemporâneo (ULHT) e Arte dos Media (ULHT/ULP). Foi professor convidado no mestrado de Artes Visuais - Intermédia da Universidade de Évora (2006-2008) e é professor convidado no mestrado em Design de Comunicação e Novos Media da Faculdade de Belas Artes da Universidade de Lisboa. A sua área de investigação centra-se na Filosofia da Comunicação, Teoria dos Media e Estética.jgomespinto@gmail.com

Carlos Pimenta é licenciado em Ciências da Comunicação - comunicação, cultura e arte (FCSH| Universidade Nova de Lisboa). Mestre em Ciências da Comunicação (Cultura Contemporânea e Novas Tecnologias - FCSH da UNL). Doutorando em Ciências da Comunicação - ECATI / ULHT. Curso de Gestão das Artes | Instituto Nacional de Administração. Curso de Fotografia (Ar.Co - Centro de Arte e Comunicação Visual). Foi Coordenador do Departamento de Teatro do IPAE (Ministério da Cultura). Foi Presidente da GDA (Sociedade de Gestão dos Direitos dos Artistas Intérpretes). Foi consultor do Instituto Camões para a internacionalização das artes. Publicou diversos artigos sobre cultura e teatro, em revistas nacionais e estrangeiras. Professor Auxiliar Convidado na Universidade Lusófona de Humanidades e Tecnologias onde é, também, assessor para as artes da ECATI e subdirector da Licenciatura em Artes Performativas e Tecnologias. Enquanto encenador dirigiu mais de duas dezenas de espectáculos de autores como Beckett, Shakespeare, Ibsen, Racine, nas principais salas do país (CCB, TNSJ, TM S. Luiz, TNDMII, etc). Desde há cerca de dez anos tem vindo a interessar-se particularmente pelas relações entre arte, cultura e tecnologia. Em 2004 foi distinguido pelo Governo Francês com o grau de Cavaleiro da Ordem das Artes e das Letras. carlosmpimenta@gmail.com 